\title{
Effect of Organic Cation Transporter 1 (OCT1) Polymorphism on Metabolic Response of Metformin in Iraqi Women with Polycystic Ovary Syndrome
}

\author{
Baraa Hussein Ali ${ }^{1}$, Ban Hoshi Khalaf ${ }^{2}$, Hassan Mahmoud Mousa AboAlmaali ${ }^{3}$ \\ ${ }^{1}$ B.Sc. Pharmacy, Candidate of Master of Science in Pharmacology and Toxicology, Department of Pharmacology \\ and Toxicology, College of Pharmacy, Kerbala University, Iraq, ${ }^{2} P h D$, Professor in Pharmacology and Toxicology, \\ Department of Pharmacology and Toxicology, College of Pharmacy, Kerbala University, Iraq, ${ }^{3}$ PhD, Assistant \\ Professor of Genetic Engineering and Biotechnology, Branch of Clinical Laboratory Sciences College of \\ Pharmacy, Kerbala University, Iraq
}

\begin{abstract}
Background: Insulin-sensitizer treatment with metformin is widely used in polycystic ovary syndrome (PCOS). However, the treatment effectiveness shows individual differences in PCOS patients. Organic cation transporter (OCT1) have been reported to mediate metformin transport in the liver. Polymorphisms of OCT1 genes may affect the activity of metformin transport and further influence the treatment response of metformin in PCOS patients.
\end{abstract}

Materials and Method: In this study, we investigated the association between the polymorphism of OCTI and the treatment effectiveness of metformin in PCOS patients. The single nucleotide polymorphism (SNPs) of OCT1 - R61C analyzed in 222 PCOS and 106 control women. Fasting serum glucose (FSG), fasting serum insulin and $\mathrm{HbAlc}$ which represented metformin treatment response, were conducted at the start of treatment and after three-month treatment.

Results: The results demonstrated that the polymorphisms of $O C T 1$ was associated with the variability of metformin response, most patients with reference allele (wild type) and heterozygous alleles of OCT1 (R61C) showed statistically significant metabolic response to metformin, while patients with mutant alleles showed less or statistically not significant response.

Conclusion: Genetic polymorphisms of $O C T 1$ contributed to different metformin treatment responses, and further study is needed to establish personalized treatment programs using a pharmacogenomic algorithm approach in PCOS patients.

Keywords: Polymorphisms, Polycystic Ovary Syndrome, Metformin, Organic cation transporter.

\section{Introduction}

Polycystic ovary syndrome (PCOS) is one of the most common endocrine disorders in women of reproductive

\section{Corresponding Author:}

\section{Baraa Hussein Ali}

B.Sc. Pharmacy, Candidate of Master of Science in Pharmacology and Toxicology, Department of Pharmacology and Toxicology, College of Pharmacy, Kerbala University, Iraq e-mail: baraaasfoor@gmail.com age. The main features include menstrual irregularity, oligoanovulation, infertility, as well as hirsutism, acne and polycystic ovarian morphology on ultrasonographic imaging $^{(1)}$. The aetiogical causation behind PCOS is yet to be precisely defined, but it is evident that familiar genetic predisposing factors interact with environmental stimuli both in utero and in pre-pubertal life $\mathrm{e}^{(2)}$.

Metformin is an off-label medication used in PCOS patients as an insulin sensitizer ${ }^{(3)}$. Metformin can not only lower elevated parameters such as insulin, androgens, and circulating free $\mathrm{T}$ levels, but can also 
increase levels of sex hormone-binding globulin (SHBG) and insulin-like growth factor-binding protein (IGFBP) (4). The well-known action of metformin is to suppress the production of hepatic glucose. Moreover, metformin has been demonstrated to increase the synthesis of SHBG and to improve menstrual frequency, ovulation, conception, and live birth rates ${ }^{(5,6)}$.

Organic cation transporter (OCT) proteins mediate the transport of organic cations across the cell membrane. Metformin has been demonstrated to be a substrate of liver-specific OCT1 and kidney-abundant OCT2 $(7,8)$. Recent studies indicated that the pharmacokinetic and pharmacodynamics profiles of metformin are mediated by the activity of OCT1 and OCT2 (9,10). In Oct1deficient mice, the hepatic metformin concentration in the liver was found to be significantly lower than that in control mice, and the glucose-lowering effects of metformin were completely abolished (11). This indicates that OCT1 expression and activity is essential for the hepatic uptake of metformin ${ }^{(12-15)}$. However, the polymorphisms of OCT1 gene may affect the activity of metformin transport and further influence the treatment response of metformin in individuals. Therefore, in this study, we investigated the association between the polymorphisms of OCT1 and the treatment effectiveness of metformin in patients with PCOS.

\section{Materials and Method}

Subjects and Study Design: In this prospective study, 222 PCOS Iraqi female patients and 106 healthy control participants were included. All patients diagnosed by consultant gynecologist according to Rotterdam criteria and treated according to practice guidelines. This study was conducted at Kerbala Teaching Hospital, Iraq, from June 2019 until April 2020 and the study protocol approved by the committee of local ethicsin the college of pharmacy, University of Kerbala, Iraq with written informed consent signed by all patients.A blood sample was collected from each overnight fasted subject in day two of the menstrual cycle and any day in case of amenorrhea in some patients for genetic,and biochemical tests) fasting serum glucose, fasting serum insulin and $\mathrm{HbAlc}$ ) before the study for all participants and after three months of treatment with metformin 500 mg twice daily for patients.

Polymerase Chain Reaction: Genomic DNAwas isolated from EDTAwhole-blood tubes with a G-DEX IIb DNA Blood Kit (Intron, Korea). Polymorphism of R61C was genotyped using Allele Specific-PCR method and specific primers were designed through Primer-BLAST, (Table 1).Optimization of PCR was recorded, (Table 2). The PCR product was run and sized by electrophoresis in the $1.5 \%$ concentration of agarose gel at $70 \mathrm{~V}$ for 60 min and visualized under Ultraviolet transmillator, the gel was photographed using a digital camera.

Table 1. Primers sequences of OCT1 rs12208357

\begin{tabular}{|l|c|c|c|}
\hline Primers & & Sequence & Product size (bp) \\
\hline \multirow{3}{*}{$\begin{array}{l}\text { Primers sequences of OCT 1 } \\
\text { (R61C) Alleles C }>\text { T }\end{array}$} & Forward & CAGATGGCCACGTGCATTCTTC & - \\
\cline { 2 - 4 } & Allele C R1 & AGGGCTCCAGCCACAGCG & 407 \\
\cline { 2 - 4 } & Allele R2 & CAGGGCTCCAGCCACAGCA & 407 \\
\hline
\end{tabular}

Table 2. PCR Condition for genotyping of OCT 1 gene (R61C) rs12208357 Alleles: C>T

\begin{tabular}{|l|c|c|c|}
\hline Steps & Temperatures/c & Time/second & Cycle \\
\hline Denature template & 94 & 3 minutes & 1 \\
\hline Initial denaturation & 94 & 30 & 30 \\
\hline Annealing & 63 & 40 & \\
\hline Extension & 72 & 55 & 1 \\
\hline Final extension & 72 & 5 minutes & \\
\hline
\end{tabular}


Data Analysis: Statistical analysis were used by software SPSS program version 20, $\mathrm{P}$-value less than 0.05 was considered a statistically significant in all gropus, Continous and Discrete variables were presented using number and percentages. Chi-square test used for comparisons of discrete variables between each study group. To compare the studied parameters within PCOS group, before and after treatment, Wilcoxon Signed Ranks Test was applied.

\section{Results and Discussion}

Metformin was the first insulin-sensitizing drug to be used in PCOS to investigate the role of insulin resistance in the pathogenesis of the syndrome. Velazquez and colleagues reported a significant improvement in menstrual regularity and reduction in circulating androgen levels in PCOS patients treated with metformin $^{(15)}$. Considerable inter-individual variability exists in response to metformin, up to one-third of patients do not respond adequately, both non-genetic and genetic factors are determinants of the metformin effect. ${ }^{(16)}$ The majority of pharmacogenetic studies performed with metformin have been focusing on the identification of gene variants related to metformin pharmacokinetics. Organic cation transporter (OCT) proteins mediate the transport of organic cations across the cell membrane. Metformin has been demonstrated to be a substrate of liver-specific OCT1, several studies indicate that the pharmacokinetic and pharmacodynamics profiles of metformin are mediated by the activity of OCT1 ${ }^{(17)}$.

Table 3 demonstrates that 222 PCOS patients and 106 healthy control enrolled in this study were at the reproductive age. Both groups in this study were overweight, BMI for PCOS patients was $31.4 \pm 4.9$ and for healthy control $27.8 \pm 4.7$. Barber et al. confirmed that weight gain and obesity occur in approximately (76\%) of women with $\mathrm{PCOS}^{(18)}$. Alopecia and hirsutism were $(92 \%)$ and $(83 \%)$ in PCOS patients, PCOS can cause both alopecia and hirsutism as one of the most reliable results of hyperandrogenism associated with this syndrome $^{(19)}$.

Genetic Analysis: Analyses were conducted to assess the association between the OCT1 polymorphism R61C (rs12208357) [CC (Reference allele), CT (heterozygous type), and TT (mutant type)] and metabolic response of metformin.

Fasting serum glucose, insulin level, $\mathrm{HbA1C}$, and HOMA-IR were significantly reduced in patients with reference alleles and heterozygous alleles but they did not in mutated alleles for R61C. Metformin exerts pleiotropic actions in several tissues, primarily the liver, where it inhibits hepatic gluconeogenesis and glycogenolysis, through which the drug contributes to improving insulin sensitivity. The potential mechanisms for inhibiting the hepatic gluconeogenesis including direct inhibition of gluconeogenic enzymes (e.g. phosphoenolpyruvate carboxykinase, fructose-1,6bisphosphatase, and glucose-6-phosphatase), reduced hepatic uptake of substrates for gluconeogenesis, and increased phosphorylation of insulin receptor and insulin receptor substrates (IRS)-1 and $-2^{(20,21)}$. Other investigators have also demonstrated the inhibition of mitochondrial respiration by metformin, may reduce the energy supply required for gluconeogenesis.

Patients with mutated alleles didn't have a significant response and this may be due to reduce or loss of OCT1 function and diminished hepatic uptake of metformin due to gene polymorphisms. Our study was compatible with Yan Shu et al. who showed that the effects of metformin on glucose tolerance tests were significantly lower in individuals carrying reduced function polymorphisms of OCT $1^{(22)}$. Sundelin et al in their study showed that hepatic distribution of metformin was significantly reduced after oral intake in carriers of 420del and R61C variants in OCT1 ${ }^{(23)}$.

Table 3. Assessment of socio-demographic data between PCOS group and healthy control group

\begin{tabular}{|l|c|c|c|}
\hline Variables & Control & PCOS & p-value \\
\hline Number & 106 & 222 & - \\
\hline Age $(\mathrm{y})$ & $28.1 \pm 6.4$ & $27.6 \pm 5.1$ & $0.451[\mathrm{NS}]$ \\
\hline BMI $\left(\mathrm{kg} / \mathrm{m}^{2}\right)$ & $27.8 \pm 4.7$ & $31.4 \pm 4.9$ & $<0.001[\mathrm{~S}]$ \\
\hline Hirsutism, $\mathrm{n}(\%)$ & $0(0.0 \%)$ & $206(92.8 \%)$ & $<0.001[\mathrm{~S}]$ \\
\hline Alopecia, $\mathrm{n}(\%)$ & $0(0.0 \%)$ & $186(83.8 \%)$ & $<0.001[\mathrm{~S}]$ \\
\hline $\begin{array}{l}\text { Results are presented as mean } \pm \mathrm{SD}, \mathrm{n}=\text { number of subjects, }(\mathrm{p}<0.05) \text { considered significantly different, [S] significant, }[\mathrm{NS}] \text { not } \\
\text { significant }\end{array}$
\end{tabular}


Table 4. Glycemic parameters in the polycystic ovary syndrome women before and after treatment with metformin according to $\mathrm{R61C}$

\begin{tabular}{|c|c|c|c|c|}
\hline Variables & Allele & Before & After & p-value \\
\hline \multirow{3}{*}{ FSG (mg/dL) } & $\mathrm{CC}$ & $97.1 \pm 13.0$ & $93.2 \pm 14.7$ & $0.001[\mathrm{~S}]$ \\
\hline & $\mathrm{CT}$ & $98.8 \pm 12.6$ & $94.6 \pm 11.7$ & $<0.001[\mathrm{~S}]$ \\
\hline & $\mathrm{TT}$ & $100.3 \pm 12.4$ & $99.7 \pm 12.7$ & $0.576[\mathrm{NS}]$ \\
\hline \multirow{3}{*}{ Insulin $(\mu \mathrm{IU} / \mathrm{ml})$} & $\mathrm{CC}$ & $22.6 \pm 14.5$ & $20.2 \pm 11.3$ & $<0.001[\mathrm{~S}]$ \\
\hline & $\mathrm{CT}$ & $23.9 \pm 15.1$ & $20.9 \pm 11.5$ & $<0.001[\mathrm{~S}]$ \\
\hline & TT & $22.8 \pm 10.6$ & $23.3 \pm 10.4$ & $0.332[\mathrm{NS}]$ \\
\hline \multirow{3}{*}{ HOMA-IR } & $\mathrm{CC}$ & $5.4 \pm 3.5$ & $4.7 \pm 2.8$ & $<0.001[\mathrm{~S}]$ \\
\hline & $\mathrm{CT}$ & $5.9 \pm 4.0$ & $5.0 \pm 2.9$ & $<0.001[\mathrm{~S}]$ \\
\hline & TT & $5.7 \pm 2.7$ & $5.8 \pm 2.7$ & $0.446[\mathrm{NS}]$ \\
\hline \multirow{3}{*}{ HbA1c (\%) } & $\mathrm{CC}$ & $4.9 \pm 0.7$ & $4.5 \pm 0.7$ & $<0.001[\mathrm{~S}]$ \\
\hline & $\mathrm{CT}$ & $4.9 \pm 0.7$ & $4.5 \pm 0.6$ & $<0.001[\mathrm{~S}]$ \\
\hline & TT & $5.6 \pm 5.4$ & $5.5 \pm 0.6$ & $0.894[\mathrm{NS}]$ \\
\hline
\end{tabular}

\section{Conclusion}

OCT1 polymorphism can be considered as one of the genetic factors responsible for heterogeneity in the metabolic response to metformin in Iraqi female suffering from PCOS. For future studies we recommend further OCT1 SNPs can be studied in order to determine the effect of multiple SNPs on response to metformin. Studying polymorphisms of genes encoding other metformin transporters such as OCT2 and OCT3. All that can lead to prescribe the right medication in precise dose for accurate duration and minimize the chance of side effects to reach the goal of therapy for polycystic ovary syndrome.

Ethical Clearance: Informed consent was obtained from all participants, Data were collected in accordance with declaration of Helsinki of the World Medical Association, 2013, all other ethical issues were approved by the authors from the University of Kerbala

Conflict of Interest: Authors Declared none.

Funding: None, self-funded by corresponding author

\section{References}

1. Escobar-Morreale HF. Polycystic ovary syndrome: definition, aetiology, diagnosis and treatment. Nature reviews Endocrinology. 2018;14:270-84
2. Norman RJ, Dewailly D, Legro RS, Hickey TE. Polycystic ovary syndrome. The Lancet. 2007;370(9588):685-97.

3. Palomba, S.; Falbo, A.; Zullo, F.; Orio, F. Evidencebased and potential benefits of metformin in the polycystic ovary syndrome: A comprehensive review. Endocr. Rev. 2009, 30, 1-50.

4. Naderpoor, N.; Shorakae, S.; de Courten, B.; Misso, M.L.; Moran, L.J.; Teede, H.J. Metformin and lifestyle modification in polycystic ovary syndrome: Systematic review and meta-analysis. Hum. Reprod. Update 2015,21, 560-574.

5. Pasquali, R.; Gambineri, A. Insulin-sensitizing agents in polycystic ovary syndrome. Eur. J. Endocrinol. 2006, 154, 763-775.

6. Morin-Papunen, L.; Rantala, A.S.; Unkila-Kallio, L.; Tiitinen, A.; Hippeläinen, M.; Perheentupa, A.; Tinkanen, H.; Bloigu, R.; Puukka, K.; Ruokonen, A.; et al. Metformin improves pregnancy and live-birth rates in women with polycystic ovary syndrome (PCOS): A multicenter, double-blind, placebo-controlled randomized trial. J. Clin. Endocrinol. Metab. 2012, 97, 1492-1500.

7. Wang, D.S.; Jonker, J.W.; Kato, Y.; Kusuhara, H.; Schinkel, A.H.; Sugiyama, Y. Involvement of organic cationtransporter 1 in hepatic and intestinal distribution of metformin. J. Pharmacol. Exp. Ther. 2002, 302, 510-515. 
8. Dresser, M.J.; Leabman, M.K.; Giacomini, K.M. Transporters involved in the elimination of drugs in the kidney: Organic anion transporters and organic cation transporters. J. Pharm. Sci. 2001, 90, 397421.

9. Zolk, O. Disposition of metformin: Variability due to polymorphisms of organic cation transporters. Ann. Med. 2012, 44, 119-129.

10. Reitman, M.L.; Schadt, E.E. Pharmacogenetics of metformin response: A step in the path toward personalizedmedicine. J. Clin. Investig. 2007, 117, 1226-1229.

11. Tzvetkov, M.V.; Vormfelde, S.V.; Balen, D.; Meineke, I.; Schmidt, T.; Sehrt, D.; Sabolic, I.; Koepsell, H.;Brockmoller, J. The effects of genetic polymorphisms in the organic cation transporters oct1, oct2, and oct3on the renal clearance of metformin. Clin. Pharmacol. Ther. 2009, 86, 299306.

12. Takane, H.; Shikata, E.; Otsubo, K.; Higuchi, S.; Ieiri, I. Polymorphism in human organic cation transportersand metformin action. Pharmacogenomics 2008, 9, 415-422.

13. Graham, G.G.; Punt, J.; Arora, M.; Day, R.O.; Doogue, M.P.; Duong, J.K.; Furlong, T.J.; Greenfield, J.R.;Greenup, L.C.; Kirkpatrick, C.M.; et al. Clinical pharmacokinetics of metformin. Clin. Pharmacokinet. 2011,50, 81-98.

14. Chen, L.; Pawlikowski, B.; Schlessinger, A.; More, S.S.; Stryke, D.; Johns, S.J.; Portman, M.A.; Chen, E.;Ferrin, T.E.; Sali, A.; et al. Role of organic cation transporter 3 (slc22a3) and its missense variants in thepharmacologic action of metformin. Pharmacogenet. Genom. 2010, 20, 687-699.

15. Velazquez E, Mendoza S, Hamer T, Sosa F, Glueck CJM. Metformin therapy in polycystic ovary syndrome reduces hyperinsulinemia, insulin resistance, hyperandrogenemia, and systolic blood pressure, while facilitating normal menses and pregnancy. 1994;43(5):647-54.

16. Graham GG, Punt J, Arora M, Day RO, Doogue MP, Duong J, et al. Clinical pharmacokinetics of metformin. 2011;50(2):81-98

17. ZolkOJAom. Disposition of metformin: variability due to polymorphisms of organic cation transporters. 2012;44(2):119-29

18. Barber T, McCarthy M, Wass J, Franks SJCe. Obesity and polycystic ovary syndrome. 2006;65(2):137-45.

19. Yasa C, Dural Ö, Bastu E, Uğurlucan FGJGO, Medicine R. Hirsutism, Acne, and Hair Loss: Management of Hyperandrogenic Cutaneous Manifestations of Polycystic Ovary Syndrome. 2017;23(2):110-9.

20. Ashokkumar N, Pari LJCca. Effect of N-benzoylD-phenylalanine and metformin on carbohydrate metabolic enzymes in neonatal streptozotocin diabetic rats. 2005;351(1-2):105-13.

21. Gunton JE, Delhanty PJ, Takahashi S-I, Baxter RCJTJoCE, Metabolism. Metformin rapidly increases insulin receptor activation in human liver and signals preferentially through insulin-receptor substrate-2. 2003;88(3):1323-32.

22. Shu Y, Sheardown SA, Brown C, Owen RP, Zhang $\mathrm{S}$, Castro RA, et al. Effect of genetic variation in the organic cation transporter 1 (OCT1) on metformin action. 2007;117(5):1422-31.

23. Sundelin E, Gormsen LC, Jensen JB, Vendelbo MH, Jakobsen S, Munk OL, et al. Genetic polymorphisms in organic cation transporter 1 attenuates hepatic metformin exposure in humans. 2017;102(5):841-8. 